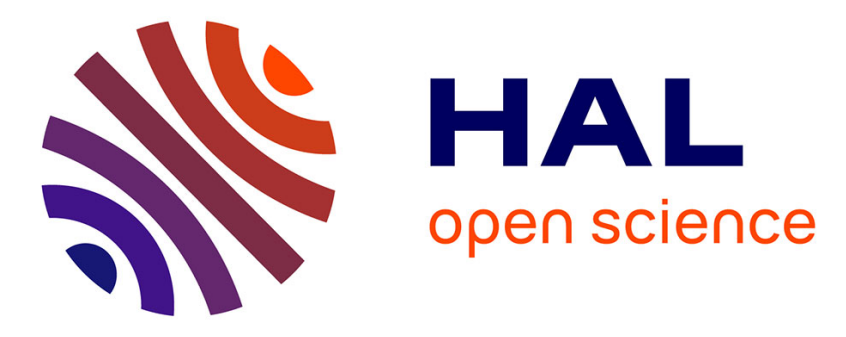

\title{
Assortative Mating in Animals and Its Role for Speciation
}

Tim Janicke, Lucas Marie-Orleach, Thomas G Aubier, Charles Perrier, Edward Morrow

\section{- To cite this version:}

Tim Janicke, Lucas Marie-Orleach, Thomas G Aubier, Charles Perrier, Edward Morrow. Assortative Mating in Animals and Its Role for Speciation. American Naturalist, 2019, 194 (6), pp.865-875. 10.1086/705825. hal-02998916

\section{HAL Id: hal-02998916 https://hal.science/hal-02998916}

Submitted on 15 Dec 2020

HAL is a multi-disciplinary open access archive for the deposit and dissemination of scientific research documents, whether they are published or not. The documents may come from teaching and research institutions in France or abroad, or from public or private research centers.
L'archive ouverte pluridisciplinaire HAL, est destinée au dépôt et à la diffusion de documents scientifiques de niveau recherche, publiés ou non, émanant des établissements d'enseignement et de recherche français ou étrangers, des laboratoires publics ou privés. 


\title{
Assortative Mating in Animals and Its Role for Speciation
}

\author{
Tim Janicke, ${ }^{1,2,3, \star}$ Lucas Marie-Orleach, ${ }^{4}$ Thomas G. Aubier, ${ }^{5}$ Charles Perrier, ${ }^{1}$ \\ and Edward H. Morrow ${ }^{2}$
}

1. Centre d'Ecologie Fonctionnelle et Evolutive, CNRS, Université de Montpellier, Université Paul Valéry Montpellier 3, École Pratique des Hautes Études, Institut de Recherche pour le Développement, 34293 Montpellier, France; 2. Evolution, Behaviour, and Environment Group, School of Life Sciences, John Maynard Smith Building, University of Sussex, Brighton BN1 9QG, United Kingdom; 3. Applied Zoology, Faculty of Biology, Technische Universität Dresden, Zellescher Weg 20b, D-01217 Dresden, Germany; 4. Centre for Biological Diversity, School of Biology, University of Saint Andrews, Saint Andrews, Fife KY16 9TH, United Kingdom; and Natural History Museum, University of Oslo, NO-0318 Oslo, Norway; and CNRS, Université de Rennes 1, ECOBIO (Ecosystèmes, Biodiversité, Evolution), Unité Mixte de Recherche 6553, F-35000 Rennes, France; 5. Department of Evolutionary Biology and Environmental Studies, University of Zurich, Winterthurerstrasse 190, CH-8057 Zurich, Switzerland

Submitted January 28, 2019; Accepted June 6, 2019; Electronically published October 9, 2019

Online enhancements: appendix. Dryad data: https://doi.org/10.5061/dryad.n445r0h.

ABSTRACT: Evolutionary theory predicts that positive assortative mating - the tendency of similar individuals to mate with each otherplays a key role for speciation by generating reproductive isolation between diverging populations. However, comprehensive tests for an effect of assortative mating on species richness at the macroevolutionary scale are lacking. We used a meta-analytic approach to test the hypothesis that the strength of assortative mating within populations is positively related to species richness across a broad range of animal taxa. Specifically, we ran a phylogenetically independent meta-analysis using an extensive database of 1,447 effect sizes for the strength of assortative mating, encompassing 307 species from 130 families and 14 classes. Our results suggest that there is no relationship between the strength of assortative mating and species richness across and within major taxonomic groups and trait categories. Moreover, our analysis confirms an earlier finding that animals typically mate assortatively (global Pearson correlation coefficient: $r=0.36$; $95 \%$ confidence interval: 0.19-0.52) when accounting for phylogenetic nonindependence. We argue that future advances will rely on a better understanding of the evolutionary causes and consequences of the observed intra- and interspecific variation in the strength of assortative mating.

Keywords: assortative mating, reproductive isolation, speciation, sexual selection.

\footnotetext{
* Corresponding author. Present address: Centre d'Ecologie Fonctionnelle et Evolutive, CNRS-Unité Mixte de Recherche 5175, 1919 Route de Mende, 34293 Montpellier, cedex 05, France; email: janicke.tim@gmail.com.

ORCIDs: Janicke, https://orcid.org/0000-0002-1453-6813; Marie-Orleach, https://orcid.org/0000-0003-3362-1500; Aubier, https://orcid.org/0000-0001-8543 -5596; Perrier, https://orcid.org/0000-0001-5820-9374; Morrow, https://orcid.org /0000-0002-1853-7469.
}

Am. Nat. 2019. Vol. 194, pp. 865-875. (C) 2019 by The University of Chicago. 0003-0147/2019/19406-59025\$15.00. All rights reserved.

DOI: $10.1086 / 705825$

\section{Introduction}

Since Darwin's (1859) pioneering work, the study of speciation - the process by which new species arise- has been at the heart of evolutionary biology. Traditionally, allopatric speciation (i.e., divergence in the presence of an extrinsic barrier that prevents genetic interchange) is often considered as the prevalent mode of speciation (Wagner 1889; Jordan 1905; Mayr 1942; reviewed in Coyne and Orr 2004). More recent work suggests that complete geographic isolation is rare, so that new species arise mainly in the presence of gene flow (Endler 1977; Gavrilets 2004) along a continuum between parapatric speciation (i.e., speciation with moderate geographic isolation and intermediate levels of gene flow) and sympatric speciation (i.e., speciation with no geographic isolation and high levels of gene flow; Butlin et al. 2008). Until now, such emergence of new species in the presence of gene flow remained a puzzling and highly controversial theme (Via 2001; Coyne and Orr 2004; Gavrilets 2014). This is because gene flow among diverging populations and associated recombination counteract divergent selection by breaking up evolving gene complexes (Felsenstein 1981). Crucially, speciation with gene flow requires a mechanism that limits recombination. Apart from the fundamental role of geographical separation, the most prominent proposed mechanism for reproductive isolation is assortative mating, which is often defined as the tendency that individuals of similar phenotypes are more likely to mate with each other than expected under random mating (Maynard Smith 1966; Kirkpatrick and Ravigné 2002).

Assortative mating has been argued to play an important role during secondary contact by promoting reproductive isolation of populations that have previously diverged in allopatry (Coyne and Orr 2004; Servedio 2016). However, a large part of theoretical work focuses on the role of assortative 
mating for speciation in sympatry or, to a lesser degree, parapatry. In a landmark review article, Kirkpatrick and Ravigné (2002) synthesized the enormous body of theoretical work on prezygotic isolation by providing a framework of five major elements that contribute to speciation. These include the source of disruptive selection, the prezygotic isolating mechanism, the way to transmit the disruptive selection to the isolating mechanism, the genetic basis of increased isolation, and the initial condition. Together with more recent work (e.g., Doebeli and Dieckmann 2003; Bolnick 2006; Kopp and Hermisson 2008; Otto et al. 2008; Thibert-Plante and Hendry 2011), it becomes evident that most models identify assortative mating as an inevitable factor for speciation in sympatry and parapatry. For a comprehensive overview of the existing literature, we refer to review articles (e.g., Weissing et al. 2011; Servedio and Boughman 2017; Kopp et al. 2018), but as a general pattern most models predict assortative mating to be a potent force generating reproductive isolation between already-diverged populations. However, for a complete understanding of the role of assortative mating for species diversity, it is also key to study whether preexisting assortative mating within genetically homogenous populations contributes to divergence. For such a scenario, most recent theoretical work amounts to the general conclusion that speciation with gene flow requires joint effects of assortative mating and disruptive selection and possibly a strong association of the loci underlying both processes (Kondrashov and Mina 1986; Gavrilets 2003, 2014; Arnegard and Kondrashov 2004; Bolnick and Fitzpatrick 2007; Servedio and Kopp 2012; Servedio 2016). Such an association can arise if there are pleiotropic effects or genetic linkage of corresponding loci (Udovic 1980) or if the trait under disruptive selection also serves as a mating signal-a phenomenon that gives rise to so-called magic traits (Gavrilets 2004; Servedio et al. 2011). Moreover, some forms of mate preferences (i.e., homotypic preferences) generate positive frequency-dependent sexual selection, meaning that individuals carrying a rare phenotype are less likely to find a (matching) mate, which then generates divergent sexual selection and favors the buildup of further assortative mating (Kirkpatrick and Nuismer 2004; Otto et al. 2008; Pennings et al. 2008).

Conversely, assortative mating has also been argued to hamper speciation. In particular, positive frequencydependent sexual selection associated with assortative mating may impede sympatric speciation by generating stabilizing selection, which eliminates polymorphism and prevents divergence (Kirkpatrick and Nuismer 2004). In a recent review, Kopp et al. (2018) highlight the importance of the underlying mating rule that gives rise to assortative mating. They argue that speciation is less likely to occur if assortative mating is associated with a "preference/trait rule" (i.e., preferences for specific traits used as mating signals) com- pared to a scenario under a matching rule (i.e., preference for matching mates). The establishment of assortative mating under a preference/trait rule requires not only the buildup of strong linkage disequilibrium between the mating signal and a diverging ecological trait but also the emergence of polymorphism at the preference locus (the so-called twoalleles mechanism; Felsenstein 1981). Unfortunately, very little is known on the type of mating rules underlying the observed assortative mating in nature (reviewed in Kopp et al. 2018).

Some of the most convincing examples for speciation with gene flow in animals come from studies on Heliconius butterflies (Insecta; Jiggins et al. 2001; Chamberlain et al. 2009; Rosser et al. 2015) and cichlid fishes (Actinopterygii; e.g., Schliewen et al. 2001; Barluenga et al. 2006). In both model systems, loci encoding for an ecological trait under disruptive selection are at least partially in physical linkage with loci serving as a mating signal, which promotes assortative mating and thereby reproductive isolation among taxa (e.g., Seehausen et al. 2008; Elmer et al. 2009; Merrill et al. 2011, 2012, 2019). Of course, speciation with gene flow often involves other isolating dimensions, such as decreased hybrid fertility, microspatial partitioning, and divergence in other ecological traits (Nosil et al. 2009; Merot et al. 2017). Yet it still raises the question of the overall contribution of assortative mating for generating the variation in species richness across the animal tree of life. If assortative mating is a precursor for speciation with gene flow to occur, we might expect a positive correlation between within-population assortative mating and species richness. However, large-scale comparative studies testing this prediction are virtually lacking to date.

In a comprehensive meta-analysis, Jiang et al. (2013) compiled more than 1,100 estimates for the strength of within-population assortative mating from a broad range of animal taxa, including 256 species from five phyla. They detected considerable variation in the strength of assortative mating among taxa and trait categories. The main outcome of this study was that animals tend to mate assortatively, based on a global effect size (measured as Pearson correlation coefficient) of $r=0.28$ that differs significantly from zero (Jiang et al. 2013). Since its publication, this study has served as a key reference for the claim that positive assortative mating is ubiquitous in the animal kingdom. Unfortunately, despite significant differences in the strength of assortative mating among phyla and lower taxa, Jiang et al. (2013) did not account for any source of phylogenetic nonindependence, as all sampled species were considered as independent data points. However, incorporating phylogenetic structure in meta-analyses has been demonstrated to be essential to obtain unbiased results (Lajeunesse 2009; Chamberlain et al. 2012; Lajeunesse et al. 2013a).

The aim of our study is therefore twofold. First, we perform a phylogenetically informed meta-analysis to assess 
the robustness of the main conclusion by Jiang et al. (2013) namely, that animals typically mate assortatively. To this end, we take stock of a database combining data sets of previously published meta-analyses with additional, more recently published estimates of assortative mating spanning a broad range of animal taxa. Second and most importantly, we provide what is to our knowledge the first test of the hypothesis that within-population assortative mating predicts species richness at the macroevolutionary scale. Our findings (i) confirm that animals show on average positive assortative mating and (ii) suggest that the strength of assortative mating is not significantly associated with species richness across and within the sampled taxa.

\section{Methods}

\section{General Approach}

We conducted a meta-analysis to test (i) whether animals show an overall positive assortative mating and (ii) whether the strength of assortative mating predicts species richness in animal taxa. In particular, we extracted estimates of the strength of assortative mating and of species richness from published data, reconstructed the phylogeny of the sampled taxa, and ran a phylogenetically independent meta-analysis.

\section{Literature Search}

We compiled estimates of within-population assortative mating from three previously published meta-analyses (Jiang et al. 2013; Graham et al. 2015; Stulp et al. 2017) and complemented this database by an additional literature screen. The vast majority of all effect sizes (1,107 estimates; $76 \%)$ were obtained from the published database by Jiang et al. (2013), who provided the hitherto most comprehensive meta-analysis of assortative mating in animals. Graham et al. (2015) studied size-assortative mating in simultaneously hermaphroditic animals, providing 33 estimates, and Stulp et al. (2017) tested for assortative mating on height in humans, providing 154 estimates. Our own additional literature search (yielding 160 estimates) followed the protocol by Jiang et al. (2013). In particular, we ran keyword searches on June 28, 2017, using the Institute for Scientific Information Web of Knowledge (Web of Science Core Collection) and Google Scholar with search terms defined as "*assortative mating," which by syntax extends the search to disassortative mating. Given that the data set by Jiang et al. (2013) spans studies published from 1923 to 2011, we screened only for studies that were published after 2011. We note that the published data set by Jiang et al. (2013) included 251 species instead of 256 as mentioned in the article. In particular, the published data file encompasses 254 species entries, of which two are erroneously listed as separate species due to inconsistent syntax (Gammarus pulex and Stercorarius parasiticus) and one due to using synonymous species names (Parus caeruleus and Cyanistes caeruleus). Furthermore, the published data set by Jiang et al. (2013) contained five effect sizes of the species Paramecium caudatum, which belongs to the Alveolata and therefore not to the animal kingdom (e.g., Adl et al. 2012; Burki 2014). Given that here we are focusing on assortative mating in animals, we excluded the family Parameciidae from our analysis. Finally, when screening the published effect sizes from the study by Jiang et al. (2013), we noticed that one effect size of the family Silphidae (Insecta) was assigned a value of one, even though the corresponding primary study does not provide any data in support of such perfect assortative mating (Knox and Scott 2006). We therefore also excluded this effect size from all analyses.

The myriad of published studies testing for assortative mating makes it virtually impossible to conduct an exhaustive literature search (already acknowledged by Jiang et al. 2013). Thus, our aim here was not to provide a complete synthesis of all published data but rather to test theory based on a wide-ranging unbiased subset of estimates for the strength of assortative mating across the animal kingdom.

\section{Data Collection}

The strength of assortative mating is typically quantified as a Pearson correlation coefficient $r$, which can be used directly as an effect size in meta-analyses (Borenstein et al. 2009). We did not transform $r$ values into Fisher's $z$ scores, as this can lead to inaccuracies in random effects metaanalysis models (reviewed in Schmidt and Hunter 2015). The sampling variance of correlation coefficients was estimated following Lajeunesse et al. (2013b). For each obtained estimate, we also recorded the reference, species, taxonomic family, and trait category for which assortative mating was tested. Specifically, traits were assigned to eight categories: age, behavior (e.g., feeding rate, dispersal, aggression), chemical compound (e.g., testosterone, pheromone), condition (e.g., residual weight for given size), genotype (e.g., heterozygosity, major histocompatibility complex alleles), ornament (e.g., color, brightness, hue), size, or structural characters (e.g., wing length, thorax length, head width).

Importantly, our study focuses exclusively on the strength of assortative mating within populations. Our database does not include experimental tests of hybridization among populations or closely related species (e.g., Culumber et al. 2014). These studies typically infer assortative mating by comparing the observed frequencies of conspecific and heterospecific matings with those expected under random mating and therefore assess whether and how strong populations are sexually isolated from one another. By excluding these data, we focus exclusively on testing whether assortative mating within populations predicts species richness within taxonomic families. 
We believe that testing this premise will inform whether assortative mating within populations promotes assortative mating among already-diverged populations and ultimately speciation. However, our study cannot inform about the relative importance of assortative mating among populations in speciation processes (Turissini et al. 2015).

There are several proposed proximate mechanisms leading to assortative mating, including sexual selection, habitat choice, host choice, and temporal/spatial covariation (Crespi 1989). Unfortunately, the vast majority of all primary studies do not provide any information about the underlying mechanism driving assortative mating in the tested model species. Therefore, we could not explore whether the effect of assortative mating on species richness depends on the mechanism responsible for assortment.

\section{Reconstruction of Phylogeny and Assessment of Species Richness}

The extracted estimates of assortative mating were obtained from a broad range of animal taxa, with the majority of effect sizes coming from amphibians, birds, insects, crustaceans, and mammals (table 1). To account for this source of statistical nonindependence (Chamberlain et al. 2012), we ran phylogenetically independent meta-analyses. We first reconstructed the phylogeny of all sampled families based on published times of divergence that were retrieved from the TimeTree database (http://www.timetree.org; Hedges et al. 2006; fig. A1; figs. A1-A4 are available online). Undated nodes were aged on the basis of the divergence times of neighboring nodes using the branch length adjuster (BLADJ) algo- rithm (Webb et al. 2008). The obtained pairwise distances were then used to compute a phylogeny using the unweighted pair group method with arithmetic mean (UPGMA) in MEGA (Kumar et al. 2018) and transformed into Newick format for further analysis (see below).

Species richness was measured as the number of known species within each of the 131 sampled families. We extracted the number of species from the Catalogue of Life database (http://www.catalogueoflife.org) on August 8, 2017. As is true for all higher taxonomic levels, family is arguably an arbitrary unit, as families may differ in the time window during which speciation could occur and older taxonomic families may contain more species. This is supported by comparative studies demonstrating that clade age rather than speciation rate predicts species richness (e.g., McPeek and Brown 2007). Therefore, the number of species may often but not always constitute a good proxy for the actual speciation rate in a given taxonomic group (Scholl and Wiens 2016). We tried to account for this potentially confounding effect by obtaining estimates of the crown age (i.e., the age of the most recent common ancestor of the extant members of the clade) for the sampled families from the TimeTree database and corrected for it statistically (see below). Estimates of crown age were available only for 103 families, which reduced the statistical power in models accounting for it.

\section{Statistical Analyses}

We studied assortative mating and its link to species richness at the family level. Therefore, we converted all individual effect sizes obtained from the same taxonomic family

Table 1: Overview of final sample sizes for the strength of assortative mating obtained per taxonomic class after excluding effect sizes from Parameciidae (Alveolata), Passerellidae (Aves), and Silphidae (Insecta)

\begin{tabular}{lrrr}
\hline Class & Effect sizes & Species & Families \\
\hline Actinopteri & 66 & 31 & 9 \\
Amphibia & 192 & 51 & 14 \\
Arachnida & 10 & 2 & 2 \\
Aves & 456 & 91 & 41 \\
Branchiopoda & 5 & 2 & 2 \\
Clitellata & 3 & 2 & 1 \\
Gastropoda & 32 & 23 & 13 \\
Insecta & 348 & 57 & 23 \\
Malacostraca & 156 & 37 & 16 \\
Mammalia & 158 & 4 & 4 \\
Merostomata & 7 & 1 & 1 \\
Reptilia & 10 & 5 & 3 \\
$\quad$ Total & 1,447 & 307 & 130 \\
\hline
\end{tabular}

Note: See "Methods." The number of effect size measures, species, and taxonomic families are shown. 
into a single mean effect size. This was done in three steps by first computing for each sampled species a mean effect size and its sampling variance for the strength of assortative mating when pooling all trait categories. These species-based effect sizes were then used to calculate a mean effect size and its sampling variance for each taxonomic genus. Finally, the same procedure was repeated for each family using the genus data. Thereby, family-based effect sizes were not biased toward species and genera with a high number of independent estimates. The family-based effect sizes were then used in the following two main analyses. First, we ran a phylogenetic independent meta-analysis to test the null hypothesis that phenotypic traits of mating partners are uncorrelated (i.e., have a mean correlation coefficient of zero) while accounting for the phylogeny using the rma.mv function of the metafor package (Viechtbauer 2010) in $\mathrm{R}$ ( $\mathrm{R}$ Core Team 2018). In this multilevel mixed effects (intercept-only) model, the effect size $r$ was fitted as a response variable weighted by the inverse of its associated sampling variance with no defined predictor variable. Phylogenetic nonindependence is accounted for by specifying the phylogenetic correlation matrix of the sampled taxonomic families as a random term. The model assumes that the effect size $r$ is normally distributed and evolves neutrally following Brownian motion. We estimated the phylogenetic signal as the phylogenetic heritability $H^{2}$ (i.e., proportional variance in $r$ explained by taxonomic family; Lynch 1991), which is equivalent to Pagel's $\lambda$ (Hansen and Orzack 2005; de Villemereuil and Nakagawa 2014). Second, we tested for a relationship between species number and assortative mating by defining log-transformed species numbers obtained for each family as a response variable and family-based mean effect sizes as a predictor variable while including the phylogenetic correlation matrix of taxonomic families as a random term. In an additional set of analyses, we added crown age as a covariate to all models to account for differences in the age of the sampled families. Finally, we provide more fine-tuned results by running both analyses separately for the major sampled taxonomic classes (i.e., those from which more than eight families had been sampled; namely, birds, insects, crustaceans, fishes, amphibians, and gastropods) and for the major trait categories (those from which more than 25 families had been sampled; namely, age, condition, ornament, size, and structural characters). Note that for all trait-specific tests, we first split the entire data set according to the five trait categories and used these subsets to compute family-based effect sizes and their sampling variance as outlined above.

The family Passerellidae (Aves) exhibited an exceptionally low mean effect size $(r=-0.85)$ arising from disassortative mating for plumage ornamentation in the whitethroated sparrow Zonotrichia albicollis, which has been detected in two independent field studies (Lowther 1961; Thorneycroft 1975). A $\chi^{2}$ test revealed that this data point should be considered as an outlier $\left(\chi^{2}=21.071, P<.001\right)$, and it was therefore excluded from all analyses (but note that including this outlier did not qualitatively change the results of any presented analysis). Together with the abovementioned exclusion criteria, this led to a final sample size of 1,447 effect sizes, including effect sizes from 307 species and 130 families (table 1). In addition to these a priori outlier tests, we ran influence diagnostics using Cook's distance $D_{i}$ and $\chi^{2}$ tests (Viechtbauer and Cheung 2010) for all models to identify outliers with a disproportionate impact on the model fit. Finally, for models with species richness as the response variable, we computed family-based effect sizes after excluding estimates of assortative mating with respect to age $(N=41)$, as this is unlikely to lead to speciation. As a consequence, models testing for effects on species richness comprised only 127 families. We did not detect signs for publication bias based on visual inspection of the funnel plot (fig. A2) or a regression test for funnel plot asymmetry $(N=130$, $z=-1.321, P=.186)$.

All statistical analyses were carried out in $\mathrm{R}$ version 3.5.1 ( $R$ Core Team 2018). Values are given as means \pm SE unless otherwise stated. All data are available in the Dryad Digital Repository (https://doi.org/10.5061/dryad.n445r0h; Janicke et al. 2019).

\section{Results}

We detected a phylogenetic signal for the strength of assortative mating of $H^{2}=0.51$, meaning that half of the observed variation among families can be attributed to the phylogeny. This highlights the necessity for phylogenetic correction in our meta-analysis. Phylogenetically independent meta-analysis (excluding the family Parameciidae) confirmed the earlier finding of a significant overall positive assortative mating within populations across animals (random effects model: $N=130, r \pm \mathrm{SE}=0.36 \pm 0.08$; $95 \%$ confidence interval: $0.19-0.52 ; z=4.223, P<.001$; fig. 1 ). This finding is also supported by the observation that 84 out of 130 sampled families (65\%) showed positive assortative mating whereas only one (1\%) and 45 (34\%) families exhibited negative or statistically nonsignificant assortative mating, respectively. Assortative mating was found to be strongest in crustaceans, gastropods, and fishes (fig. 1) but did not differ significantly among trait categories, as indicated by widely overlapping 95\% confidence intervals (fig. A3).

Species richness was not significantly related to the strength of assortative mating across all families and traits (multilevel mixed effects model: $N=127$, slope $\pm \mathrm{SE}=$ $0.25 \pm 0.30, z=0.852, P=.394$; fig. 2 ). This lack of a statistically significant effect of assortative mating on species richness was consistent across the major taxonomic classes and trait categories (table 2; fig. A4). The only exception was the Malacostraca, which showed a significant positive 


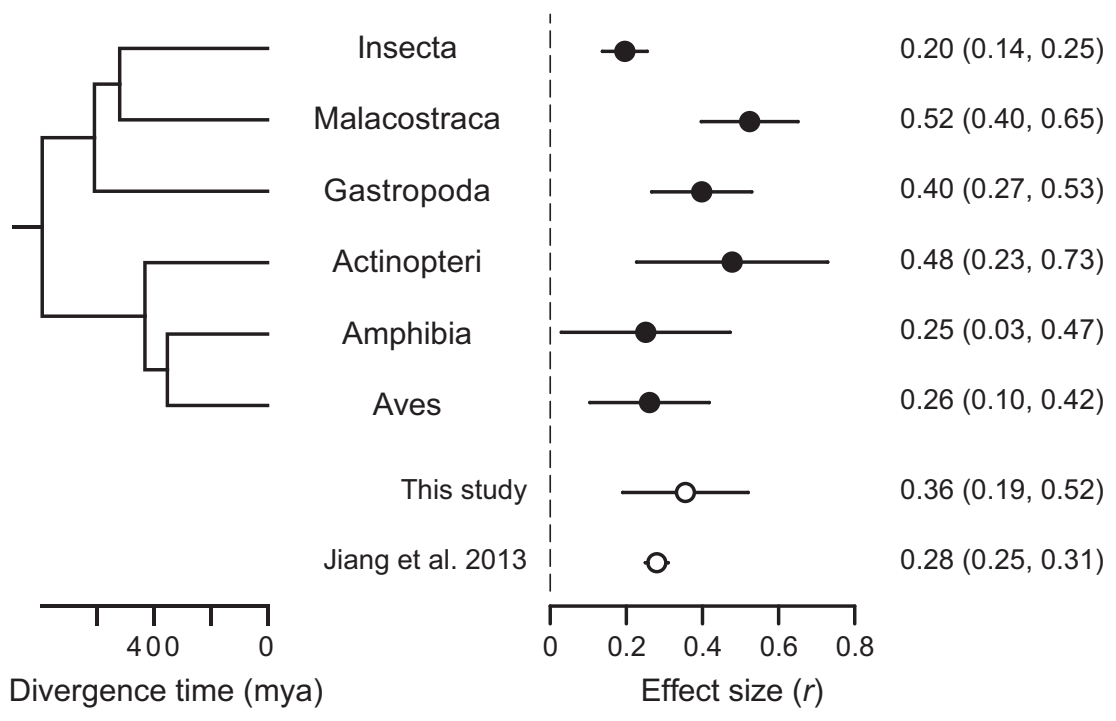

Figure 1: Phylogenetic tree and forest plot of mean effect sizes (filled circles with error bars) for the strength of within-population assortative mating shown for the main taxonomic classes included in this study. Open circles indicate global effect sizes of this study and a previous meta-analysis by Jiang et al. (2013). Error bars show $95 \%$ confidence intervals.

relationship between species richness and assortative mating. However, this class included the family Inachidae, which showed the strongest assortative mating among all families and had a disproportionate influence on the model outcome (Cook's distance: $D_{i}=0.19 ; \chi^{2}=6.545, P=.011$ ). When excluding this family from the analysis, the positive relationship within the Malacostraca became statistically nonsignificant (multilevel mixed effects model: $N=15$, $\left.r \pm \mathrm{SE}=0.86 \pm 0.69, Q_{\mathrm{M}}=1.261, P=.207\right)$. In an analysis accounting for interfamily variation in crown age including all sampled taxa, we again found no significant relationship between species number and the strength of assortative mating (multilevel mixed effects model: $N=103$; crown age: slope $\pm \mathrm{SE}=0.003 \pm 0.002, z=1.516, P=.130$; assortative mating: slope $\pm \mathrm{SE}=-0.186 \pm 0.294, z=-0.634$, $P=.526)$ and also no significant relationship within taxonomic classes or trait categories (table A1, available online).

\section{Discussion}

Our results clearly demonstrate that the mean strength of assortative mating within populations is not related to species richness at the family level across a broad range of animal taxa and therefore do not support the hypothesis that within-population assortative mating predicts the enormous variation in species richness in the animal tree of life. Moreover, our study confirms an earlier finding of a prevalence of positive assortative mating in animals. Below we discuss both of these findings as well as the limitations of our study.

\section{Assortative Mating and Speciation}

Our study provides no support for the hypothesis that withinpopulation assortative mating increases the probability for speciation. We found that half of the observed variation in the strength of assortative mating can be attributed to the

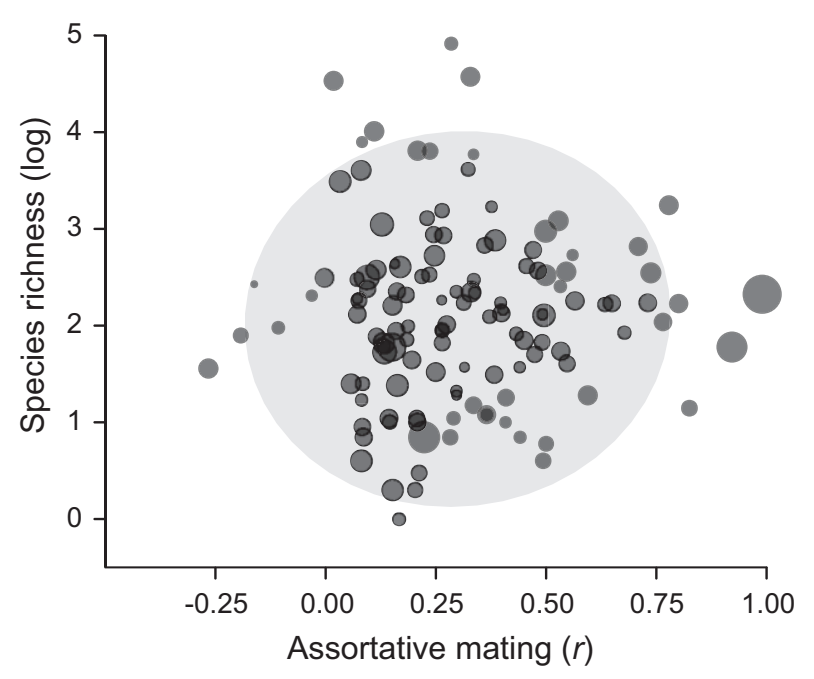

Figure 2: Relationship between species richness and the strength of within-population assortative mating. Each data point represents the effect size and the log-transformed number of species of a given family. The size of each data point corresponds to the precision of its effect size (i.e., the log-transformed reciprocal of its sampling variance). The shaded area indicates the $95 \%$ confidence ellipse. 
Table 2: Phylogenetically independent meta-analysis testing for a relationship between species richness and the strength of within-population assortative mating for the major taxonomic classes and trait categories

\begin{tabular}{lrcrrr}
\hline Level and subset & $N$ & Slope $\pm \mathrm{SE}$ & $z$ & $P$ & $q$ \\
\hline Class: & & & & & \\
$\quad$ Birds & 39 & $-.251 \pm .457$ & -.55 & .583 & .662 \\
Insects & 23 & $.702 \pm 1.603$ & .44 & .662 & .662 \\
Malacostraca & 16 & $1.148 \pm .427$ & 2.69 & .007 & .043 \\
Fishes & 9 & $.877 \pm 1.500$ & .58 & .559 & .662 \\
Amphibians & 14 & $-.860 \pm .686$ & -1.26 & .210 & .419 \\
Gastropods & 13 & $1.383 \pm .753$ & 1.84 & .066 & .198 \\
Trait category: & & & & & \\
Condition & 25 & $-.659 \pm .454$ & -1.45 & .147 & .586 \\
Ornament & 31 & $-.134 \pm .435$ & -.31 & .758 & .883 \\
Size & 112 & $.206 \pm .286$ & .72 & .472 & .883 \\
Structural trait & 52 & $-.062 \pm .420$ & -.15 & .883 & .883 \\
\hline
\end{tabular}

Note: $N$ refers to the number of sampled families. $q$ values refer to $P$ values corrected for false discovery rate following Benjamini and Hochberg (1995). Boldfacing indicates statistically significant effects at $\alpha<.05$.

phylogeny, meaning that assortative mating differed substantially among the sampled phylogenetic units. But despite this phylogenetic signal, the observed strength of assortative mating was unrelated to the number of described species within families.

This lack of a relationship between assortative mating and species richness may result from a relatively minor contribution of speciation with gene flow to the generation of species richness. In an attempt to estimate the importance of sympatric speciation over allopatric speciation, Bolnick and Fitzpatrick (2007) found that $9.4 \%$ of 309 described speciation events resulted in sister species with more than $90 \%$ range overlap, suggesting that sympatric speciation is indeed rare compared to allopatric speciation. However, the authors point to several limitations of their approach, including problems of identifying sister species and the difficulty of quantifying the range overlap at the time of speciation (Bolnick and Fitzpatrick 2007). Importantly, assortative mating not only is involved in sympatric or parapatric speciation but has also been argued to constitute an important mechanism in maintaining reproductive isolation after secondary contact between allopatrically diverged populations via reinforcement (e.g., Kondrashov and Shpak 1998; Noor 1999; Servedio and Noor 2003). Taxa showing positive within-population assortative mating are more likely to remain reproductively isolated after secondary contact if they diverged for the same trait that underlies assortative mating. Under such a secondary-contact scenario, we would also predict a positive relationship between assortative mating and species richness, which we did not detect in our study.

Another reason for the absence of a link between assortative mating and species richness might be that the conditions under which within-population assortative mating promotes speciation are often not fulfilled. The most prominent of these conditions is strong linkage of the loci coding for mating signals with loci under disruptive selection (Udovic 1980; Gavrilets 2003). Unfortunately, information regarding the prevalence of such linkage is currently unavailable for the vast majority of species covered by our meta-analysis. Similarly, we also lack data on the prevalence of "magic traits" (e.g., ecological adaptation traits that also serve as mating signals; Gavrilets 2004). In general, magic traits have been argued to be more widespread than originally thought (Servedio 2011; Servedio and Kopp 2012). Interestingly, Servedio et al. (2011) provide a list of putatively good examples for magic traits that is dominated by morphological traits such as body size, structural traits, and ornaments. These are also the most frequent trait categories in our meta-analysis. However, this concordance does not imply that the majority of the traits included in our metaanalysis are actually magic traits, as it may simply reflect an overall bias toward studying these particular traits. Therefore, it is impossible to assess whether the observed lack of a relationship between assortative mating and species richness is the consequence of generally rare linkage between genes under disruptive selection and those that encode for mating signals.

The lack of an overall relationship between assortative mating and species richness might also be explained by interspecific variation in the underlying mating rule. Mating rules (constituting either one- or two-allele mechanisms sensu Felsenstein 1981) have been argued to differ drastically in their likelihood of affecting speciation with gene flow (reviewed by Kopp et al. 2018). Under a preference/trait mating rule, reproductive isolation requires not only strong linkage disequilibrium between loci under disruptive selection and 
those encoding for mating signals but also the evolution of polymorphism at the preference locus (i.e., two-allele mechanism), which renders speciation intrinsically more difficult compared to a matching rule scenario (often constituting a one-allele mechanism). Given that the preference/trait mating rule is generally thought to be widespread in nature, assortative mating may not necessarily be associated with increased speciation rate. However, as pointed out by Kopp et al. (2018), we currently lack estimates of the prevalence of this mating rule, as such estimates require extensive work in both the field and the laboratory.

An additional explanation for the absence of a relationship between assortative mating and species richness is that strong assortative mating may actually constrain rather than promote speciation. Specifically, certain forms of assortative mating cause stabilizing sexual selection, because rare phenotypes are less likely to find mating partners compared to common phenotypes. Theoretical models demonstrate that such underlying positively frequency-dependent selection may erode genetic variation and weaken the linkage disequilibrium between loci, which both underlie the genetic basis of sympatric speciation (e.g., Kirkpatrick and Nuismer 2004). Moreover, other theoretical work suggests that very strong assortative mating may suppress trait differentiation among populations after secondary contact (Servedio 2011; Servedio and Burger 2014; reviewed in Servedio 2016). All these arguments question the view that within-population assortative mating is a factor that always favors speciation (Gavrilets 2003).

Methodological limitations of our study might have also obscured the effect of assortative mating on species richness. As a general feature of almost all meta-analyses, we may have included effect sizes that are not fully comparable. In our particular case, the extracted estimates for the strength of assortative mating may result from different biological processes. There are many mechanisms that can give rise to assortative mating, of which sexual selection involving mating preferences is only one of them (reviewed in Crespi 1989). Notably, individuals with similar phenotypes are often temporally or spatially clustered in terms of phenotypically plastic responses to environmental conditions, which may lead to assortative mating in the absence of mate choice simply because mating pairs were sampled across the entire population, ignoring this temporal and spatial heterogeneity (Fernández-Meirama et al. 2017). Even if this type of assortative mating of individuals living in the same microhabitat is not based on mating preferences, it can still be critical to speciation because it could be effective in preventing gene flow (e.g., speciation by host choice in tephritid fruit flies of the genus Rhagoletis; Bush 1969). We are aware of only a few studies of assortative mating that assessed such temporal and spatial segregation of the studied population and accounted for it statistically. These studies suggest that estimates of the strength of assortative mating can largely be driven by spatial clustering of similar phenotypes rather than mate choice (Pal et al. 2006; Graham et al. 2015; Ng et al. 2016).

Finally, the absence of a relationship between assortative mating and species richness may also arise if reproductive isolation has only a minor effect on speciation rate at the macroevolutionary scale. Specifically, diversification rates might be limited primarily by ecological factors or mechanisms that promote range expansion and the formation of allopatric isolates rather than reproductive isolation itself (e.g., Price 2010; Gillman and Wright 2014). In accordance with this, Rabosky and Matute (2013) demonstrated that the rate at which birds and fruit flies evolve reproductive isolation is decoupled from the rate at which they form new species.

We note that our results do not refute the view that sexual selection affects species richness (e.g., Janicke et al. 2018; but see Kraaijeveld et al. 2011). This is because sexual selection (defined as selection arising from competition for mating partners and/or for fertilizing the partner's gametes; Shuker 2010) can impact species richness either by preventing species from extinction or by promoting speciation of which assortative mating is only one possible mechanism among others (reviewed in Ritchie 2007; Servedio and Boughman 2017). Moreover, sexual selection is only one mechanism that may lead to assortative mating within a population (Crespi 1989). Therefore, the lack of a relationship between assortative mating and species richness does not mean that sexual selection does not relate to species richness.

\section{Reassessment of Assortative Mating in Animals}

The second aim of our study was to provide a more robust test of the hypothesis that assortative mating is on average positive. Our phylogenetically informed meta-analysis revealed a global effect size that is positive and statistically different from zero, supporting the main finding of an early meta-analysis by Jiang et al. (2013), which did not account for phylogenetic nonindependence. However, we obtained a larger global effect size with five times broader confidence intervals (effect size $r=0.36$; 95\% confidence interval: 0.19 0.52 ) compared to the study by Jiang et al. (2013; effect size $r=0.28$; 95\% confidence interval: $0.25-0.31$ ), suggesting that assortative mating is much more variable between taxa than previously thought. This result is in line with a previously documented higher risk of type I error in metaanalyses that do not correct for phylogenetic nonindependence. Chamberlain et al. (2012) reanalyzed 30 published meta-analyses to compare results obtained from traditional (i.e., not controlling for phylogeny) and phylogenetically independent meta-analyses. Their results indicate that accounting for a phylogenetic signal leads to a heterogeneity 
that is on average nine times larger, which translates into larger confidence limits and often affects whether the obtained global effect sizes differ significantly from zero (Chamberlain et al. 2012).

Statistical correction for phylogenetic relationships is widely established in a large variety of comparative studies to overcome problems arising from nonindependence of data from related taxa. Nonetheless, more recently phylogenetic correction has been argued to be misleading, especially when studying discrete characters that arise from singular evolutionary events and in the absence of a phylogenetic signal in the studied trait (Uyeda et al. 2018). Given that we were studying a continuous trait with a strong phylogenetic signal, we believe that our phylogenetically independent meta-analysis is not confounded by these issues. However, our analysis may still have some limitations, as it relies on the phylogeny used and the assumed model of evolution. While we are confident that our phylogenetic tree of taxonomic families accurately reflects phylogenetic affinities (at least in terms of the topology), we admit that our model of evolution (Brownian motion) might be an oversimplification. Bearing this in mind, we are still convinced that accounting for the phylogenetic signal (even when based on an oversimplified model of evolution) is a favorable approach for metaanalyses that cover different taxa, as is the case for classic comparative studies.

\section{Conclusions}

We show that assortative mating within populations does not predict species richness in animals. Empirical evidence for often-made assertions such as that assortative mating constitutes a "fundamental process which generates biodiversity on our planet" (Ingley and Rosenthal 2017) is still lacking at a global scale. Despite methodological limitations, our meta-analysis supports the idea that within-population assortative mating alone does not promote speciation and/ or that the conditions under which it does are not widespread enough to explain species richness across animals. Our study also corroborates the view that positive assortative mating is widespread. Future work should aim at a better understanding of the evolutionary causes and consequences of the observed intra- and interspecific variation in the strength of assortative mating.

\section{Acknowledgments}

We thank Mike Ritchie for helpful comments on an earlier draft. This study was funded by a research grant from the Deutsche Forschungsgemeinschaft to T.J. (Temporary Positions for Principal Investigators module; grant JA 2653/ 2-1), a fellowship of the Swiss National Science Foundation to L.M.-O. (grant P300PA_171516), and a Royal Society University Research Fellowship to E.H.M.

\section{Literature Cited}

Adl, S. M., A. G. Simpson, C. E. Lane, J. Lukeš, D. Bass, S. S. Bowser, M. W. Brown, et al. 2012. The revised classification of eukaryotes. Journal of Eukaryotic Microbiology 59:429-514.

Arnegard, M. E., and A. S. Kondrashov. 2004. Sympatric speciation by sexual selection alone is unlikely. Evolution 58:222-237.

Barluenga, M., K. N. Stölting, W. Salzburger, M. Muschick, and A. Meyer. 2006. Sympatric speciation in Nicaraguan crater lake cichlid fish. Nature 439:719-723.

Benjamini, Y., and Y. Hochberg. 1995. Controlling the false discovery rate - a practical and powerful approach to multiple testing. Journal of the Royal Statistical Society B 57:289-300.

Bolnick, D. I. 2006. Multi-species outcomes in a common model of sympatric speciation. Journal of Theoretical Biology 241:734-744.

Bolnick, D. I., and B. M. Fitzpatrick. 2007. Sympatric speciation: models and empirical evidence. Annual Review of Ecology, Evolution, and Systematics 38:459-487.

Borenstein, M., L. V. Hedges, J. P. T. Higgins, and H. R. Rothstein. 2009. Introduction to meta-analysis. Wiley, Chichester.

Burki, F. 2014. The eukaryotic tree of life from a global phylogenomic perspective. Cold Spring Harbor Perspectives in Biology 6:a016147.

Bush, G. L. 1969. Sympatric host race formation and speciation in frugivorous flies of the genus Rhagoletis (Diptera, Tephritidae). Evolution 23:237-251.

Butlin, R. K., J. Galindo, and J. W. Grahame. 2008. Sympatric, parapatric or allopatric: the most important way to classify speciation? Philosophical Transactions of the Royal Society B 363:2997-3007.

Chamberlain, N. L., R. I. Hill, D. D. Kapan, L. E. Gilbert, and M. R. Kronforst. 2009. Polymorphic butterfly reveals the missing link in ecological speciation. Science 326:847-850.

Chamberlain, S. A., S. M. Hovick, C. J. Dibble, N. L. Rasmussen, B. G. Van Allen, B. S. Maitner, J. R. Ahern, et al. 2012. Does phylogeny matter? assessing the impact of phylogenetic information in ecological meta-analysis. Ecology Letters 15:627-636.

Coyne, J. A., and H. A. Orr. 2004. Speciation. Sinauer, Sunderland, MA.

Crespi, B. J. 1989. Causes of assortative mating in arthropods. Animal Behaviour 38:980-1000.

Culumber, Z. W., O. M. Ochoa, and G. G. Rosenthal. 2014. Assortative mating and the maintenance of population structure in a natural hybrid zone. American Naturalist 184:225-232.

Darwin, C. R. 1859. On the origin of species by means of natural selection. J. Murray, London.

de Villemereuil, P., and S. Nakagawa. 2014. General quantitative genetic methods for comparative biology. Pages 287-303 in L. Z Garamszegi, ed. Modern phylogenetic comparative methods and their application in evolutionary biology. Springer, Berlin.

Doebeli, M., and U. Dieckmann. 2003. Speciation along environmental gradients. Nature 421:259-264.

Elmer, K. R., T. K. Lehtonen, and A. Meyer. 2009. Color assortative mating contributes to sympatric divergence of Neotropical cichlid fish. Evolution 63:2750-2757.

Endler, J. A. 1977. Geographic variation, speciation, and clines. Monographs in Population Biology 10. Princeton University Press, Princeton, NJ. 
Felsenstein, J. 1981. Skepticism towards Santa Rosalia, or why are there so few kinds of animals? Evolution 35:124-138.

Fernández-Meirama, M., D. Estévez, T. Ng, G. A. Williams, A. Carvaja-Rodríguez, and E. Rolán-Alvarez. 2017. A novel method for estimating the strength of positive mating preference by similarity in the wild. Ecology and Evolution 7:2883-2893.

Gavrilets, S. 2003. Models of speciation: what have we learned in 40 years? Evolution 57:2197-2215.

. 2004. Fitness landscapes and the origin of species. Princeton University Press, Princeton, NJ.

. 2014. Models of speciation: where are we now? Journal of Heredity 105:743-755.

Gillman, L. N., and S. D. Wright. 2014. Species richness and evolutionary speed: the influence of temperature, water and area. Journal of Biogeography 41:39-51.

Graham, S., E. Chapuis, S. Meconcelli, N. Bonel, K. Satori, A. Christophe, P. Alda, P. David, and T. Janicke. 2015. Size-assortative mating in simultaneous hermaphrodites: an experimental test and a meta-analysis. Behavioral Ecology and Sociobiology 69:1867-1878.

Hansen, T. F., and S. H. Orzack. 2005. Assessing current adaptation and phylogenetic inertia as explanations of trait evolution: the need for controlled comparisons. Evolution 59:2063-2072.

Hedges, S. B., J. Dudley, and S. Kumar. 2006. TimeTree: a public knowledge-base of divergence times among organisms. Bioinformatics 22:2971-2972.

Ingley, S. J., and G. G. Rosenthal. 2017. Mechanisms of assortative mating and ecological speciation. Evolution 71:185-186.

Janicke, T., L. Marie-Orleach, T. G. Aubier, C. Perrier, and E. H. Morrow. 2019. Data from: Assortative mating in animals and its role for speciation. American Naturalist, Dryad Digital Repository, https://doi.org/10.5061/dryad.n5445r5060h.

Janicke, T., M. G. Ritchie, E. H. Morrow, and L. Marie-Orleach. 2018. Sexual selection predicts species richness across the animal kingdom. Proceedings of the Royal Society B 285:20180173.

Jiang, Y. X., D. I. Bolnick, and M. Kirkpatrick. 2013. Assortative mating in animals. American Naturalist 181:E125-E138.

Jiggins, C. D., R. E. Naisbit, R. L. Coe, and J. Mallet. 2001. Reproductive isolation caused by colour pattern mimicry. Nature 411:302-305.

Jordan, D. S. 1905. The origin of species through isolation. Science 22:545-562.

Kirkpatrick, M., and S. L. Nuismer. 2004. Sexual selection can constrain sympatric speciation. Proceedings of the Royal Society B 271:687-693.

Kirkpatrick, M., and V. Ravigné. 2002. Speciation by natural and sexual selection: models and experiments. American Naturalist 159(suppl.):S22-S35.

Knox, T. T., and M. P. Scott. 2006. Size, operational sex ratio, and mate-guarding success of the carrion beetle, Necrophila americana. Behavioral Ecology 17:88-96.

Kondrashov, A. S., and M. V. Mina. 1986. Sympatric speciation: when is it possible? Biological Journal of the Linnean Society 27:201-223.

Kondrashov, A. S., and M. Shpak. 1998. On the origin of species by means of assortative mating. Proceedings of the Royal Society B 265:2273-2278.

Kopp, M., and J. Hermisson. 2008. Competitive speciation and costs of choosiness. Journal of Evolutionary Biology 21:1005-1023.

Kopp, M., M. R. Servedio, T. C. Mendelson, R. J. Safran, R. L. Rodríguez, M. E. Hauber, E. C. Scordato, et al. 2018. Mechanisms of assortative mating in speciation with gene flow: connecting theory and empirical research. American Naturalist 191:1-20.
Kraaijeveld, K., F. J. L. Kraaijeveld-Smit, and M. E. Maan. 2011. Sexual selection and speciation: the comparative evidence revisited. Biological Reviews 86:367-377.

Kumar, S., G. Stecher, M. Li, C. Knyaz, and K. Tamura. 2018. MEGA $\mathrm{X}$ : molecular evolutionary genetics analysis across computing platforms. Molecular Biology and Evolution 35:1547-1549.

Lajeunesse, M. J. 2009. Meta-analysis and the comparative phylogenetic method. American Naturalist 174:369-381.

Lajeunesse, M. J., M. S. Rosenberg, and M. D. Jennions. 2013a. Phylogenetic nonindependence and meta-analysis. Pages 284-299 in J. Koricheva, J. Gurevitch, and K. Mengersen, eds. Handbook of meta-analysis in ecology and evolution. Princeton University Press, Princeton, NJ.

- 2013b. Recovering missing or partial data from studies: a survey of conversions and imputations for meta-analysis. Pages 195206 in J. Koricheva, J. Gurevitch, and K. Mengersen, eds. Handbook of meta-analysis in ecology and evolution. Princeton University Press, Princeton, NJ.

Lowther, J. K. 1961. Polymorphism in the white-throated sparrow, Zonotrichia albicollis (Gmelin). Canadian Journal of Zoology 39:281292.

Lynch, M. 1991. Methods for the analysis of comparative data in evolutionary biology. Evolution 45:1065-1080.

Maynard Smith, J. 1966. Sympatric speciation. American Naturalist 100:637-650.

Mayr, E. 1942. Systematics and the origin of species from the viewpoint of a zoologist. Columbia University Press, New York.

McPeek, M. A., and J. M. Brown. 2007. Clade age and not diversification rate explains species richness among animal taxa. American Naturalist 169:E97-E106.

Merot, C., C. Salazar, R. M. Merrill, C. D. Jiggins, and M. Joron. 2017. What shapes the continuum of reproductive isolation? lessons from Heliconius butterflies. Proceedings of the Royal Society B 284:20170335.

Merrill, R. M., Z. Gompert, L. M. Dembeck, M. R. Kronforst, W. O. McMillan, and C. D. Jiggins. 2011. Mate preference across the speciation continuum in a clade of mimetic butterflies. Evolution 65:1489-1500.

Merrill, R. M., P. Rastas, S. H. Martin, M. C. Melo, S. Barker, J. Davey, W. O. McMillan, and C. D. Jiggins. 2019. Genetic dissection of assortative mating behavior. PLoS Biology 17:e2005902.

Merrill, R. M., R. W. Wallbank, V. Bull, P. C. Salazar, J. Mallet, M. Stevens, and C. D. Jiggins. 2012. Disruptive ecological selection on a mating cue. Proceedings of the Royal Society B 279:4907-4913.

Ng, T. P. T., G. A. Williams, M. S. Davies, R. Stafford, and E. RolanAlvarez. 2016. Sampling scale can cause bias in positive assortative mating estimates: evidence from two intertidal snails. Biological Journal of the Linnean Society 119:414-419.

Noor, M. A. F. 1999. Reinforcement and other consequences of sympatry. Heredity 83:503-508.

Nosil, P., L. J. Harmon, and O. Seehausen. 2009. Ecological explanations for (incomplete) speciation. Trends in Ecology and Evolution 24:145-156.

Otto, S. P., M. R. Servedio, and S. L. Nuismer. 2008. Frequencydependent selection and the evolution of assortative mating. Genetics 179:2091-2112.

Pal, P., J. Erlandsson, and M. Skold. 2006. Size-assortative mating and non-reciprocal copulation in a hermaphroditic intertidal limpet: test of the mate availability hypothesis. Marine Biology 148:12731282 . 
Pennings, P. S., M. Kopp, G. Meszéna, U. Dieckmann, and J. Hermisson. 2008. An analytically tractable model for competitive speciation. American Naturalist 171:E44-E71.

Peters, J. L., A. J. Sutton, D. R. Jones, K. R. Abrams, and L. Rushton. 2008. Contour-enhanced meta-analysis funnel plots help distinguish publication bias from other causes of asymmetry. Journal of Clinical Epidemiology 61:991-996.

Price, T. D. 2010. The roles of time and ecology in the continental radiation of the Old World leaf warblers (Phylloscopus and Seicercus). Philosophical Transactions of the Royal Society B 365:1749-1762.

Rabosky, D. L., and D. R. Matute. 2013. Macroevolutionary speciation rates are decoupled from the evolution of intrinsic reproductive isolation in Drosophila and birds. Proceedings of the National Academy of Sciences of the USA 110:15354-15359.

R Core Team. 2018. R: a language and environment for statistical computing. R Foundation for Statistical Computing, Vienna. Version 3.5.1.

Ritchie, M. G. 2007. Sexual selection and speciation. Annual Review of Ecology, Evolution, and Systematics 38:79-102.

Rosser, N., K. M. Kozak, A. B. Phillimore, and J. Mallet. 2015. Extensive range overlap between heliconiine sister species: evidence for sympatric speciation in butterflies? BMC Evolutionary Biology 15:125.

Schliewen, U., K. Rassmann, M. Markmann, J. Markert, T. Kocher, and D. Tautz. 2001. Genetic and ecological divergence of a monophyletic cichlid species pair under fully sympatric conditions in Lake Ejagham, Cameroon. Molecular Ecology 10:1471-1488.

Schmidt, F. L., and J. E. Hunter. 2015. Methods of meta-analysis: correcting error and bias in research findings. Sage, London.

Scholl, J. P., and J. J. Wiens. 2016. Diversification rates and species richness across the tree of life. Proceedings of the Royal Society B 283:20161334.

Seehausen, O., Y. Terai, I. S. Magalhaes, K. L. Carleton, H. D. Mrosso, R. Miyagi, I. van der Sluijs, et al. 2008. Speciation through sensory drive in cichlid fish. Nature 455:620-626.

Servedio, M. R. 2011. Limits to the evolution of assortative mating by female choice under restricted gene flow. Proceedings of the Royal Society B 278:179-187.

-2016. Geography, assortative mating, and the effects of sexual selection on speciation with gene flow. Evolutionary Applications 9:91-102.

Servedio, M. R., and J. W. Boughman. 2017. The role of sexual selection in local adaptation and speciation. Annual Review of Ecology, Evolution, and Systematics 48:85-109.

Servedio, M. R., and R. Burger. 2014. The counterintuitive role of sexual selection in species maintenance and speciation. Proceedings of the National Academy of Sciences of the USA 111:8113-8118.
Servedio, M. R., and M. Kopp. 2012. Sexual selection and magic traits in speciation with gene flow. Current Zoology 58:510-516.

Servedio, M. R., and M. A. F. Noor. 2003. The role of reinforcement in speciation: theory and data. Annual Review of Ecology, Evolution, and Systematics 34:339-364.

Servedio, M. R., G. S. Van Doorn, M. Kopp, A. M. Frame, and P. Nosil. 2011. Magic traits in speciation: "magic" but not rare? Trends in Ecology and Evolution 26:389-397.

Shuker, D. M. 2010. Sexual selection: endless forms or tangled bank? Animal Behaviour 79:E11-E17.

Stulp, G., M. J. Simons, S. Grasman, and T. V. Pollet. 2017. Assortative mating for human height: a meta-analysis. American Journal of Human Biology 29:e22917.

Thibert-Plante, X., and A. P. Hendry. 2011. Factors influencing progress toward sympatric speciation. Journal of Evolutionary Biology 24:2186-2196.

Thorneycroft, H. B. 1975. A cytogenetic study of the white-throated sparrow, Zonotrichia albicollis (Gmelin). Evolution 29:611-621.

Turissini, D. A., G. Liu, J. R. David, and D. R. Matute. 2015. The evolution of reproductive isolation in the Drosophila yakuba complex of species. Journal of Evolutionary Biology 28:557-575.

Udovic, D. 1980. Frequency-dependent selection, disruptive selection, and the evolution of reproductive isolation. American Naturalist 116:621-641.

Uyeda, J. C., R. Zenil-Ferguson, and M. W. J. S. B. Pennell. 2018. Rethinking phylogenetic comparative methods. Systematic Biology 67:1091-1109.

Via, S. 2001. Sympatric speciation in animals: the ugly duckling grows up. Trends in Ecology and Evolution 16:381-390.

Viechtbauer, W. 2010. Conducting meta-analyses in $\mathrm{R}$ with the metafor package. Journal of Statistical Software 36:1-48.

Viechtbauer, W., and M. W. L. Cheung. 2010. Outlier and influence diagnostics for meta-analysis. Research Synthesis Methods 1:112125.

Wagner, M. 1889. Die Entstehung der Arten durch räumliche Sonderung. B. Schwabe, Basel.

Webb, C. O., D. D. Ackerly, and S. W. Kembel. 2008. Phylocom: software for the analysis of phylogenetic community structure and trait evolution. Bioinformatics 24:2098-2100.

Weissing, F. J., P. Edelaar, and G. S. van Doorn. 2011. Adaptive speciation theory: a conceptual review. Behavioral Ecology and Sociobiology 65:461-480. 